\title{
Efficient numerical solution to vacuum decay with many fields
}

\author{
Ali Masoumi, ${ }^{1}$ Ken D. Olum, ${ }^{1}$ and Benjamin Shlaer ${ }^{1,2}$ \\ ${ }^{1}$ Institute of Cosmology, Department of Physics and Astronomy, Tufts University, Medford, \\ MA 02155, USA \\ ${ }^{2}$ Department of Physics, University of Auckland, Private Bag 92019, Auckland, New Zealand \\ E-mail: ali@cosmos.phy.tufts.edu,kdo@cosmos.phy.tufts.edu, \\ shlaer@cosmos.phy.tufts.edu
}

\begin{abstract}
Finding numerical solutions describing bubble nucleation is notoriously difficult in more than one field space dimension. Traditional shooting methods fail because of the extreme non-linearity of field evolution over a macroscopic distance as a function of initial conditions. Minimization methods tend to become either slow or imprecise for larger numbers of fields due to their dependence on the high dimensionality of discretized function spaces. We present a new method for finding solutions which is both very efficient and able to cope with the non-linearities. Our method directly integrates the equations of motion except at a small number of junction points, so we do not need to introduce a discrete domain for our functions. The method, based on multiple shooting, typically finds solutions involving three fields in around a minute, and can find solutions for eight fields in about an hour. We include a numerical package for Mathematica which implements the method described here.
\end{abstract}




\section{Contents}

1 Introduction $\quad 1$

$2 \quad O(4)$ bounce formalism $\quad 2$

3 Solution techniques 3

4 Description of the method 5

4.1 Multiple shooting method 5

4.2 Analytic solutions 7

$\begin{array}{lll}\text { 4.2.1 Analytic solution for large } r & 7\end{array}$

4.2.2 Analytic solutions near the center of the bounce 8

4.3 Adding a new variable 8

4.4 Rescaling $\quad 9$

4.5 Readjusting $r$ values $r$

4.6 Choice of the initial profile 10

4.7 Calculation of the action 11

5 Examples of potentials solved by the code $\quad 12$

6 Quality of the code 13

6.1 Range of application 13

6.2 Success fraction 13

$\begin{array}{lll}6.3 \text { Speed } & 14\end{array}$

6.4 Quality of solution 14

6.5 Possible issues 14

7 Comparison with other methods $\quad 15$

8 Conclusion $\quad 15$

$\begin{array}{ll}\text { A Calculation of Jacobian } & 16\end{array}$

$\begin{array}{lll}\text { A.1 Solving for Jacobian along the path } & 16\end{array}$

$\begin{array}{lll}\text { A.2 Jacobian with respect to } \Delta & 17\end{array}$

$\begin{array}{ll}\text { B Powell's method for solving equations } & 18\end{array}$

$\begin{array}{lr}\text { C Obtaining and running the code } & 19\end{array}$

\section{Introduction}

The thermal or quantum mechanical nucleation of bubbles initiates a first-order phase transition. This phenomenon has application in diverse areas of physics including superfluid Helium-3 [1, 2], Higgs-induced vacuum decay, [3-7], baryogenesis and gravitational waves from a first-order electroweak phase transition [8-14], and quantum gravitational instabilities $[13,15,16]$. The idea that the observable universe represents but one vacuum $[17,18]$ 


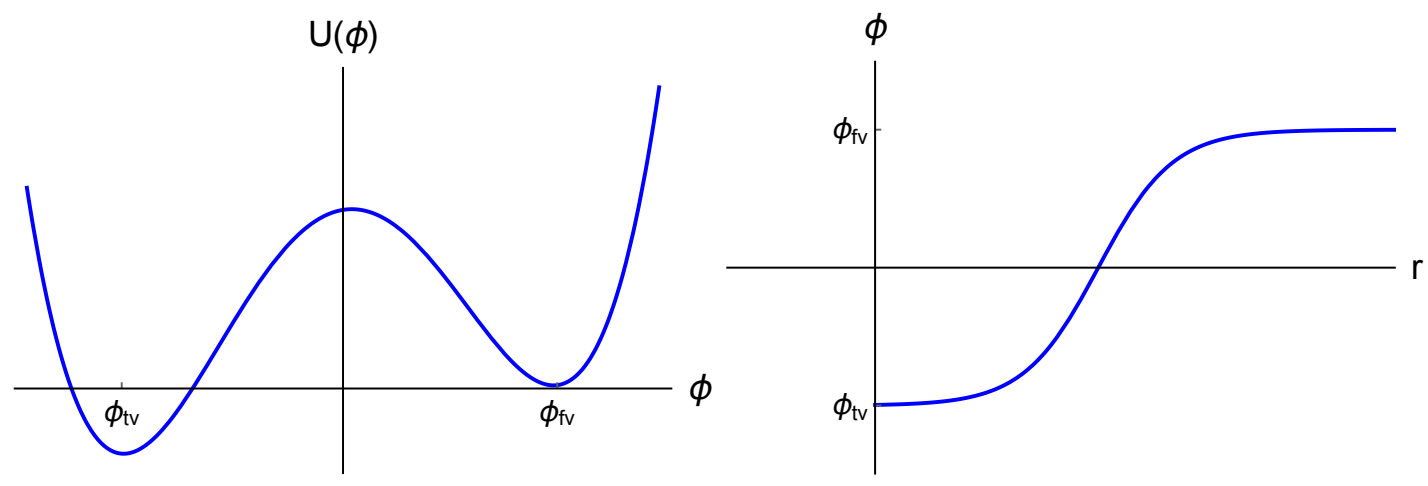

Figure 1. Left panel shows a potential with two vacua. The left vacuum $\varphi_{\mathrm{tv}}$ is stable and denoted as the "true vacuum." The right vacuum $\varphi_{\mathrm{fv}}$, which is meta-stable, is called the "false vacuum." Right panel shows the field profile that carries the tunneling.

in a diverse landscape of string vacua $[19,20]$ motivates a study of false vacuum decay in high-dimensional field spaces [21-28].

Quantum field theories with an effective potential $U(\phi)$ have perturbatively stable vacua corresponding to the local minima of $U(\phi)$. Non-perturbative effects can destabilize these local minima via the spontaneous nucleation of bubbles containing a lower energy perturbative vacuum [29]. Instanton methods [30, 31] provide a simple and elegant treatment of this process. The primary calculation is a Euclidean $O(4)$-symmetric "bubble" field profile (called the bounce) which smoothly interpolates between a true-vacuum configuration at its center and the false vacuum whose decay is being described. Once this is calculated, the decay rate follows, as well as the field configuration for the formation and evolution of a single bubble.

This paper is structured as follows. We briefly review the bounce formalism in section 2 . In section 3 we describe simple algorithms for finding the bounce solution and the problems they face. In section 4 we describe our more robust method for finding the bounce. In section 5 we give some simple examples, and in section 6 we describe the abilities of our code. In section 7 we compare our method with other methods, and we conclude in section 8 .

\section{$2 O(4)$ bounce formalism}

In this section we follow Coleman [30]. We start from the Lagrangian for a scalar field in flat space,

$$
\mathcal{L}=\frac{1}{2} \partial_{\mu} \phi \partial^{\mu} \phi-U(\phi)
$$

where $U(\phi)$ is a potential with two minima. An example is shown in Figure 1. The tunneling is described by a solution to the Euclidean $\left(x_{0} \rightarrow-i \tau\right)$ equations of motion,

$$
\nabla^{2} \phi=\frac{\partial U}{\partial \phi}
$$

Assuming that the field has an $O(4)$ symmetry, i.e., $\phi(x)=\phi(r)$ where $r=\sqrt{\tau^{2}+\sum x_{i}^{2}}$, (2.2) simplifies to

$$
\frac{d^{2} \phi}{d r^{2}}+\frac{D-1}{r} \frac{d \phi}{d r}=\frac{\partial U}{\partial \phi}
$$


where $D$ is the number of spacetime dimensions. This is the equation of motion for a particle that moves in the "upside-down" potential $-U(\phi)$ under the influence of the velocitydependent "friction" term $(D-1) / r$. The boundary conditions are

$$
\phi^{\prime}(0)=0, \quad \lim _{r \rightarrow \infty} \phi(r)=\varphi_{\mathrm{fv}},
$$

where the prime denotes differentiation with respect to $r$.

It is the friction that allows the field to roll to a stop atop the false-vacuum hill, which is lower than the true-vacuum initial position. Since friction weakens inversely with the time parameter $r$, beginning too close to the peak of the true-vacuum hill will result in a large initial delay, and so too little friction when velocities are large; the field will overshoot the false vacuum.

The simplest non-constant solution with the above properties is known as the bounce. A typical potential and the corresponding bounce field profile are shown in Figure 1. The tunneling rate is given by $\Gamma=A e^{-B}$, where $B$ is the Euclidean action of the corresponding bounce minus the action of the false vacuum configuration.

Generalization of this problem to more than one field is straightforward. We denote the fields by $\phi=\left(\phi_{1}, \phi_{2}, \ldots, \phi_{N}\right)$ and the potential by $U(\phi)$. The bounce is then a solution to the system of coupled ordinary differential equations

$$
\frac{d^{2} \phi_{i}}{d r^{2}}+\frac{D-1}{r} \frac{d \phi_{i}}{d r}=\frac{\partial U}{\partial \phi_{i}}
$$

with boundary conditions $d \phi /\left.d r\right|_{r=0}=0$ and $\lim _{r \rightarrow \infty} \phi(r)=\varphi_{\mathrm{fv}}$. This is a system of $N$ second-order equations with $N$ boundary conditions at $r=0$ and another $N$ at $r=\infty$. The field must begin at $r=0$ from some specific point at the center of the bubble and asymptotically approach the false vacuum infinitely far away. Finding the solution to the bounce equations is tantamount to determining the values of the fields at $r=0$.

For a single field, Coleman [30] used an overshoot-undershoot argument to prove that there is always a bounce that connects a higher meta-stable vacuum to an adjacent lower energy vacuum. However, with more than one field there are cases where no solution exists, as shown, for example, in figure 2.

There are also cases that have more than one solution. With more than one field, there may be more than one "mountain pass" connecting the two vacua, as in figure 7 below. Each pass gives rise to its own instanton. Even with only one field, there are cases with multiple solutions. One example is shown in figure 3, where we drew the upside down potential. Regions shown in red overshoot the true vacuum, while those shown in black undershoot. Thus the right edge of the upper red region and the two edges of the lower red region are each a solution.

For a thermally induced first-order phase transition the formalism is quite similar [1, 31].

\section{Solution techniques}

The most straightforward method to find the bounce numerically would be shooting from the initial condition $\phi(0)=\varphi_{0}$. Starting from some guess for $\varphi_{0}$, we could integrate the equations of motion to large $r$. The correct value of $\varphi_{0}$ yields the desired asymptotic boundary condition $\phi \rightarrow \varphi_{\mathrm{fv}}$. We could iteratively improve $\varphi_{0}$ based on how close $\phi$ comes to $\varphi_{\mathrm{fv}}$.

This technique requires integrating the differential equation (2.5) from $r=0$ to large $r$. For a single field, so long as we are descending the inverted potential from the true vacuum, 


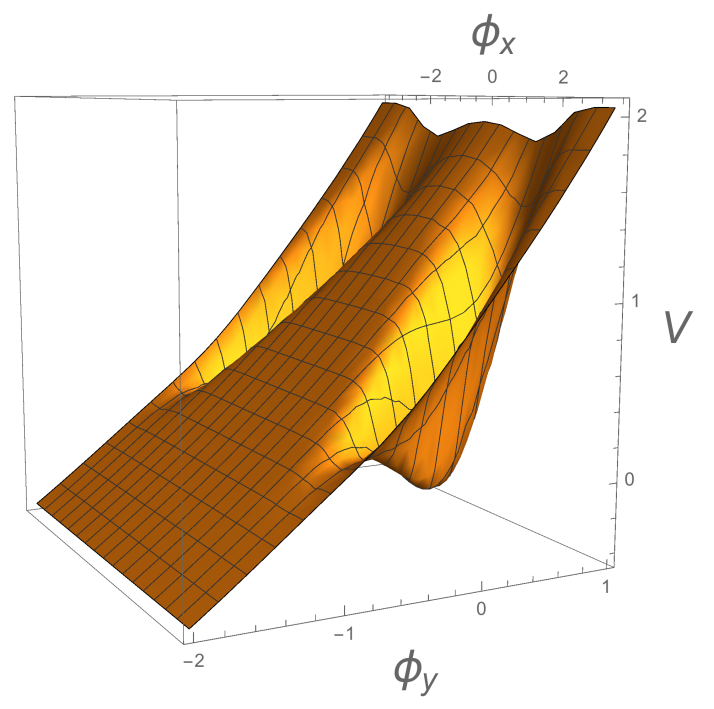

Figure 2. In this two-dimensional potential there is no instanton connecting the two minima on the right. The problem is that tunneling between the two minima is dominated by tunneling to lower values on the left.

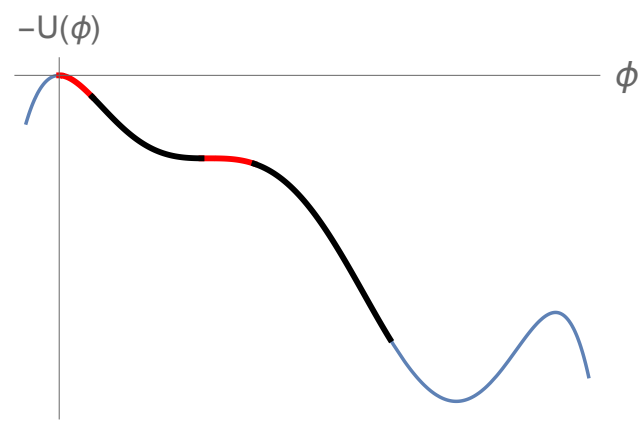

Figure 3. A one-dimensional potential, shown inverted, that has more than one solution.

this equation is stable. But as we ascend the inverted potential toward the false vacuum, it becomes unstable. The desired solution asymptotically approaches $\varphi_{\mathrm{fv}}$, but there is another solution which more and more rapidly falls down the hill. This growing mode causes numerical instability that makes it impossible to find the solution with the correct $\phi \rightarrow \varphi_{\mathrm{fv}}$ boundary condition.

This problem can be avoided by a "double shooting" scheme [32]. We pick $\phi_{0}$ as above and pick also some large but finite value $r_{\max }$ and a field value $\phi\left(r_{\max }\right)=\varphi_{\max }$ there. Then we integrate (2.3) to the right from $r=0$ and to the left from $r_{\max }$, and demand that the two solutions match at some intermediate location. We then attempt to iteratively improve $\phi_{0}$ and $\varphi_{\max }$.

The double shooting technique works reasonably well for one field, but for additional fields we face additional problems. One problem is that the solution may travel along a "ridge" in the inverted potential. In this case, there is an unstable mode that falls off the ridge instead of traveling along it. Reversing the direction of integration does not help this problem.

This problem exists even when the field is descending the inverted potential. Unless it is following the steepest descent, modes that descend in steeper directions will always be more 
unstable than the desired mode. This occurs even near the true and near the false vacuum.

A related problem is that in many cases, $\varphi_{0}$ can never be specified with sufficient accuracy. ${ }^{1}$ For example, consider a potential which near the true vacuum has the form

$$
U\left(\phi_{1}, \phi_{2}\right)=10\left(\phi_{1}^{2}+\phi_{2}^{2}\right)-16 \phi_{1} \phi_{2}
$$

Neglecting friction, the solution is

$$
\begin{aligned}
& \phi_{1}=A e^{2 r}+B e^{6 r}, \\
& \phi_{2}=A e^{2 r}-B e^{6 r} .
\end{aligned}
$$

Suppose we want to reach $\phi=(2,0)$ at $r=10$. Then we need $A=e^{-20}, B=e^{-60}$, and thus $\varphi_{0}=\left(e^{-20}+e^{-60}, e^{-20}-e^{-60}\right)$. But in double-precision floating point, $e^{-20} \pm e^{-60}$ is indistinguishable from $e^{-20}$, so we end up with $\varphi_{0}=\left(e^{-20}, e^{-20}\right)$, and thus $\phi(10)=$ $(1,1)$ instead of $(2,0)$. Including the friction term would lead to Bessel functions instead of exponentials, but the effect is the same.

\section{Description of the method}

As mentioned in section 2, the simple shooting methods for solving the bounce equations face several difficulties, which we address here. The most significant problem is solving a set of differential equations that are unstable. We overcome this problem using a multi-interval shooting method described in subsection 4.1. At the center of the bubble $(r=0)$ there is a divergence in (2.5). Additionally, the other boundary condition is set at $r=\infty$, which cannot be represented as a floating-point number. We solve these problems by using an approximate analytic solution in subsection 4.2. In subsection 4.3 we add an additional variable to the set for which we are solving, in order to make it easier for the system to change the solution by translation in $r$, in subsection 4.4 we rescale the potential so that all function values used in the solution have similar ranges, and in subsection 4.5 we make changes to the division points between the shooting regions.

To find the bounce solution using shooting methods we need to come up with an initial guess. The procedure for finding a good initial guess is described in subsection 4.6. After we find the solution, we need to calculate the action, which is discussed in subsection 4.7.

Solving non-linear equations using steepest decent guarantees that the steps taken toward the solution are small, but this method converges slowly. On the other hand Newton's method converges rapidly, but can take dangerously large steps. We use Powell's hybrid method, which converges quickly near the solution, yet takes reasonable steps far away from it. This is explained in appendix B. To use the this method we need to calculate the Jacobian of the field values with respect to the initial conditions. We use an accurate method for calculating the Jacobian which is described in appendix A. Appendix C explains how to download and run the code.

\subsection{Multiple shooting method}

The differential equations (2.5) are unstable in the sense that there are growing and decaying modes, and the growing modes may have different rates of growth. Unless we are interested

\footnotetext{
${ }^{1}$ The one-field version of this problem is discussed by Wainwright [33], who solves it by using the logarithm of the distance from the true vacuum as a parameter.
} 
in the fastest mode, a small admixture of this mode from numerical error will eventually overpower the solution of interest. To avoid this problem, we integrate the differential equations only over a range of $r$ small enough that the growth is tolerable, and put together several such regions to cover the needed range of $r$. This method is known as multiple shooting [34].

To understand the degree of instability, let us expand $U(\phi)$ around some specific point $\varphi_{1}$ to quadratic order,

$$
U(\phi)=U\left(\boldsymbol{\varphi}_{1}\right)+A_{i}\left(\phi-\varphi_{1}\right)_{i}+\left(B_{i j} / 2\right)\left(\phi-\varphi_{1}\right)_{i}\left(\phi-\varphi_{1}\right)_{j}+\mathcal{O}\left(\phi-\varphi_{1}\right)^{3},
$$

where

$$
A_{i}=\left.\frac{\partial U}{\partial \phi_{i}}\right|_{\varphi_{1}}, \quad B_{i j}=\left.\frac{\partial^{2} U}{\partial \phi_{i} \partial \phi_{j}}\right|_{\varphi_{1}} .
$$

If we use (4.2) and ignore the frictional term in (2.5), we have

$$
\frac{d^{2} \phi_{i}}{d r^{2}}=A_{i}+B_{i j}\left(\phi-\varphi_{1}\right)_{j}
$$

We can diagonalize the symmetric matrix $B_{i j}$ using an orthogonal matrix $O$, so $B_{i j}=$ $O_{k i} \tilde{B}_{k l} O_{l j}$ with $\tilde{B}_{k l}=\operatorname{diag}\left(B_{1}, B_{2}, \ldots, B_{N}\right)$. Define $\tilde{\phi}_{i}=O_{i j}\left(\phi-\varphi_{1}\right)_{j}$ and $\tilde{A}_{i}=O_{i j} A_{j}$, so that

$$
\frac{d^{2} \tilde{\phi}_{i}}{d r^{2}}=\tilde{A}_{i}+B_{i} \tilde{\phi}_{i}
$$

Assuming that all $B_{i}$ are positive, which is the case near the true and false vacua, but not everywhere, the solutions to (4.4) include a mode that grows as $e^{\sqrt{B_{i}}}$ and one that shrinks as $e^{-\sqrt{B_{i}}}$. If $B_{\max }$ is the largest of the $B_{i}$, then the worst case is that the correct solution goes as $e^{-\sqrt{B_{\max }}}$, so the fastest-growing mode grows relative to the correct solution as $e^{2 \sqrt{B_{\max }}}$.

Let the domain $r \in[0, \infty)$ be divided by $n \geq 3$ intermediate points, $\left\{r_{1}, r_{2}, \ldots, r_{n}\right\}$. We discuss the method of choosing these points in subsection 4.6. At each step, our variables are the values of the field at $r_{1}$ and $r_{n}$ and for $n>3$ the field and its derivative at $r_{2}, \ldots, r_{n-2}$. Notice that we do not use the value of the field and its derivative at $r_{n-1}$ as variables. As described in the next subsection, we do not solve the differential equation for $r<r_{1}$ nor $r>r_{N}$. Instead, in these regions, we approximate the potential by a quadratic and so get an analytic solution for $\phi(r)$, and using this solution we determine $\boldsymbol{\phi}^{\prime}\left(r_{1}\right)$ and $\boldsymbol{\phi}^{\prime}\left(r_{n}\right)$. Having the field and derivatives we can do the following numerical integrations of (2.5):

1. Integrate from $r_{i}$ to $r_{i+1}$ for $i=1 \ldots n-2$ to obtain $\phi_{L}\left(r_{i+1}\right)$ and $\phi_{L}^{\prime}\left(r_{i+1}\right)$.

2. Integrate from $r_{n}$ to $r_{n-1}$ to $\phi_{R}\left(r_{n-1}\right)$ and $\phi_{R}^{\prime}\left(r_{n-1}\right)$.

Here $\phi_{L}$ and $\phi_{R}$ denote the values of these quantities obtained by integrating from the left and from the right, respectively. Since we want a smooth solution to the equations of motion, we try to match the values of $\phi_{L}$ and $\phi_{L}^{\prime}$ with the corresponding variables at $r_{2}, r_{3}, \ldots r_{n-2}$ and $\phi_{L}$ and $\phi_{L}^{\prime}$ with $\phi_{R}$ and $\phi_{R}^{\prime}$ at point $r_{n-1}$. This is shown for $n=3$ and $n=4$ in Figure 4.

The problem is now reduced to finding the values of $(2 n-4) N$ variables that satisfy $(2 n-4) N$ equations. We use Powell's hybrid method, described briefly in appendix B, for this purpose.

The reason for shooting toward the left in the last interval is that in this interval (at least by $r_{n}$ ) the inverted potential is increasing toward the false vacuum. Shooting forward 

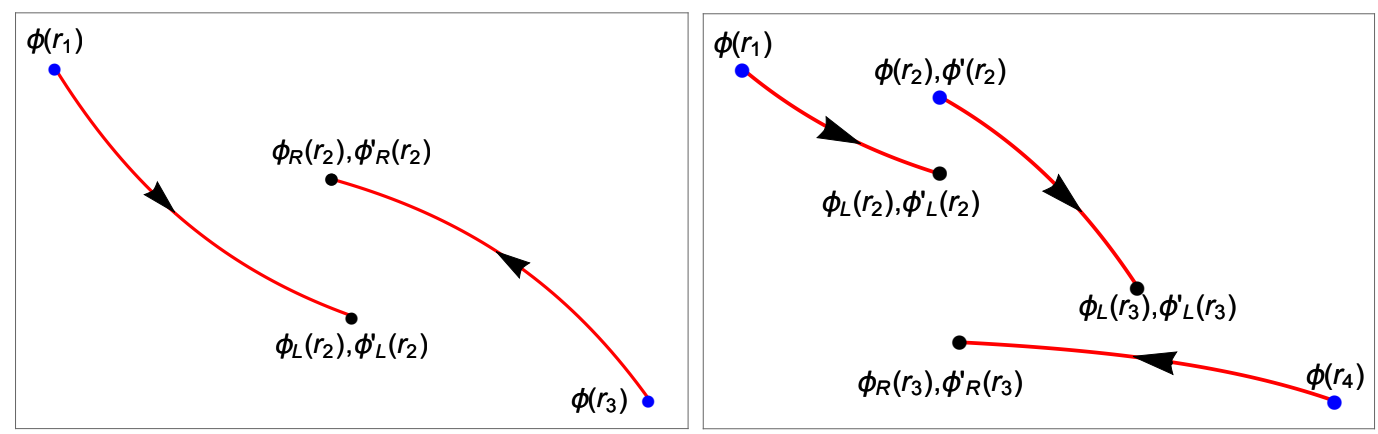

Figure 4. The left panel shows the parameters used when there are only three points. We try to find $\phi\left(r_{1}\right)$ and $\phi\left(r_{3}\right)$ to make $\phi_{L}\left(r_{2}\right)=\phi_{R}\left(r_{2}\right)$ and $\phi_{L}^{\prime}\left(r_{2}\right)=\phi_{R}^{\prime}\left(r_{2}\right)$. The right panel shows the case of four points. Here we try to find $\phi\left(r_{1}\right), \phi\left(r_{2}\right), \phi^{\prime}\left(r_{2}\right)$, and $\phi\left(r_{4}\right)$ to make $\phi_{L}\left(r_{2}\right)=\phi\left(r_{2}\right), \phi_{L}^{\prime}\left(r_{2}\right)=$ $\phi^{\prime}\left(r_{2}\right), \phi_{L}\left(r_{3}\right)=\phi_{R}\left(r_{3}\right)$, and $\phi_{L}^{\prime}\left(r_{3}\right)=\phi_{R}^{\prime}\left(r_{3}\right)$.

corresponds to going uphill which is unstable in proportion to the largest eigenvalue around the false vacuum, but shooting downhill is only sensitive to the difference between eigenvalues, and hence we reduce the sensitivity of the solution. A possible improvement would be to shoot toward the left for more intervals if the potential is also increasing there, but we don't have any way to know in advance which these will be, and so we did not implement this technique.

\subsection{Analytic solutions}

As described above, we use closed-form solutions to equations with an approximate potential for $r<r_{1}$ and $r>r_{N}$. This technique solves the problem where finite numerical accuracy makes it impossible to solve the equations of motion at $\phi=\varphi_{0}$, and another problem where if we integrate the differential equation for sufficiently large $r$, numerical inaccuracy will always lead to a divergence.

\subsubsection{Analytic solution for large $r$}

When the field is very close to the false vacuum, we can approximate $U(\phi)$ with a quadratic. This allows an approximate analytic solution for $\phi$. Of the two solutions to the second order field equations, we clearly want the one which approaches a constant as $r \rightarrow \infty$. This enables us to deduce $\phi^{\prime}\left(r_{n}\right)$ as a function of $\phi\left(r_{n}\right)$. We then use $\phi\left(r_{n}\right)$ and $\phi^{\prime}\left(r_{n}\right)$ to integrate backward to $r_{n-1}$ and apply the matching conditions there.

So consider $r_{n}$ large enough so that the fields $\phi\left(r_{n}\right)$ are very close to their false vacuum values. Unless the field has the correct derivative, it will fall off the hill in some direction; for a given $\phi\left(r_{n}\right)$ there is a unique $\phi^{\prime}\left(r_{n}\right)$ that lands on the hilltop. The potential is given by the expansion (4.1) around $\varphi_{\mathrm{fv}}$, with $A_{i}=0$ here, and the equation of motion becomes

$$
\frac{d^{2} \tilde{\phi}_{i}}{d r^{2}}+\frac{D-1}{r} \frac{d \tilde{\phi}_{i}}{d r}=B_{i} \tilde{\phi}_{i}
$$

We are interested in the solution which approaches $\tilde{\phi}_{i}(r)=0$ as $r \rightarrow \infty$, which is

$$
\tilde{\phi}_{i}(r)=C_{i} r^{\frac{2-D}{2}} K_{\frac{D-2}{2}}\left(\sqrt{B_{i}} r\right)
$$


where $K_{(D-2) / 2}$ denotes the Bessel function of type $K$, and the $C_{i}$ are constants. Returning to the original field variables,

$$
\phi_{i}(r)=\varphi_{\mathrm{fv} i}+\sum_{j} O_{j i} C_{j} r^{\frac{2-D}{2}} K_{\frac{D-2}{2}}\left(\sqrt{B_{j}} r\right) .
$$

For a given $\phi\left(r_{n}\right)$ we determine the $C_{i}$ and from there calculate $\boldsymbol{\phi}^{\prime}\left(r_{n}\right)$, which we use to shoot towards $r_{n-1}$.

\subsubsection{Analytic solutions near the center of the bounce}

The situation near $r=0$ is similar. We do not use $\varphi_{0}$ as a parameter, but instead use $\varphi_{1}=\phi\left(r_{1}\right)$. Here we do not assume that $\varphi_{1}$ is close to the true vacuum, and we expand not around $\varphi_{\mathrm{tv}}$ but around $\varphi_{1}$, which is accurate as long as the $\varphi_{0}$ that we infer is not too far from $\varphi_{1}$.

The expansion is exactly as in (4.1), and the resulting equation of motion is

$$
\frac{d^{2} \tilde{\phi}_{i}}{d r^{2}}+\frac{D-1}{r} \frac{d \tilde{\phi}_{i}}{d r}=\tilde{A}_{i}+B_{i} \tilde{\phi}_{i}
$$

The initial condition $\tilde{\phi}_{i}^{\prime}(0)=0$ picks out the solution with $I$-type Bessel functions,

$$
\tilde{\phi}_{i}(r)=C_{i} r^{\frac{2-D}{2}} I_{\frac{D-2}{2}}\left(\sqrt{B_{i}} r\right)-\frac{\tilde{A}_{i}}{B_{i}}
$$

and the requirement that $\phi\left(r_{1}\right)=\varphi_{1}$, so $\tilde{\phi}_{i}\left(r_{1}\right)=0$, fixes the $C_{i}$, giving

$$
\tilde{\phi}_{i}(r)=\frac{\tilde{A}_{i}}{B_{i}}\left[\left(\frac{r_{1}}{r}\right)^{\frac{D-2}{2}} \frac{I_{\frac{D-2}{2}}\left(r \sqrt{B_{i}}\right)}{I_{\frac{D-2}{2}}\left(r_{1} \sqrt{B_{i}}\right)}-1\right] .
$$

In the original field variables,

$$
\phi_{j}(r)=\phi_{1 j}+\sum_{j} O_{j i} \frac{\tilde{A}_{j}}{B_{j}}\left[\left(\frac{r_{1}}{r}\right)^{\frac{D-2}{2}} \frac{I_{\frac{D-2}{2}}\left(r \sqrt{B_{j}}\right)}{I_{\frac{D-2}{2}}\left(r_{1} \sqrt{B_{j}}\right)}-1\right] .
$$

Thus from $\varphi_{1}$ we can find the derivative $\phi^{\prime}\left(r_{1}\right)$, and we use these to shoot to $r_{2}$.

\subsection{Adding a new variable}

A generic field profile of the correct bounce solution (e.g., figure 1) has plateaus at small and large $r$ and a relatively small transition region. However, we do not have any a priori knowledge of the $r$ values near the transition. This leads to an important numerical difficulty. The differential equations in (2.5) exhibit an approximate $r$-translation symmetry that is broken by the friction term. If $\phi(r)$ is a solution, $\phi(r+\Delta)$ would almost satisfy the differential equation and we will be in a situation depicted in Figure 5. If the code finds a translated version of the field profile, it nearly satisfies the constraints. While the two profiles are simply related by translation in $r$, in the field variables used in the solution, $\phi\left(r_{1}\right), \phi\left(r_{2}\right), \phi^{\prime}\left(r_{1}\right), \ldots$ the translation is very complicated, requiring adjustment of all variables in a coordinated way. This makes it difficult for the code to make progress after finding the translated profile. A similar issue was discussed in Ref. [35] 


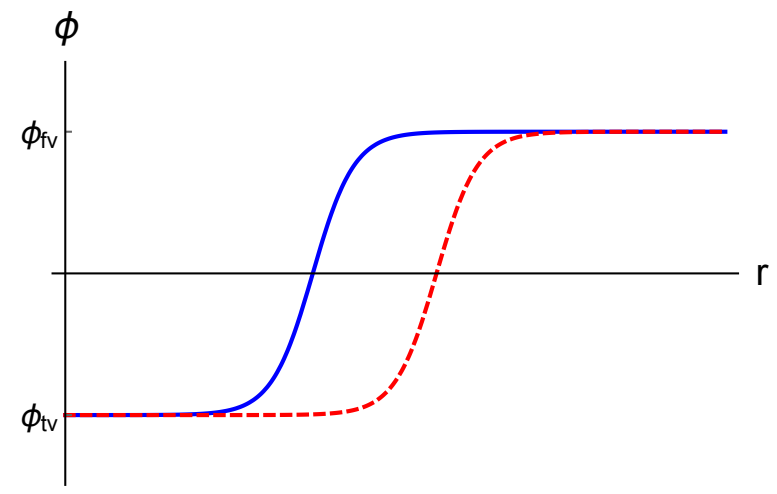

Figure 5. Correct (solid blue) and translated (dashed red) field profiles. If the code finds the translated profile, it may be difficult for it to find the correct profile.

To solve this problem, we add to the variables used by the equation solver an additional variable $\Delta$, representing a translation of the parameters $r_{i}$. Instead of using the set of points $r_{1}, r_{2}, \ldots, r_{n}$ shown in Figure 4 we use the set of $r_{1}+\Delta, r_{2}+\Delta, \ldots, r_{n}+\Delta$. Thus changing $\Delta$ does not change the values of the fields and derivative variables, but changes the location where these variables are applied, making it simple for the solver to move in the direction of translations in $r$.

By introducing $\Delta$, we have one more unknown than our equations, so there are infinitely many solutions, but any one is the solution to the problem, expressed in its own way. This method proves to be very effective and extends the range of potentials for which the code can find solutions. This technique is discussed further in Ref. [36].

\subsection{Rescaling}

The bounce solution has a simple transformation under rescaling of the fields and the potential. If the potential $U(\phi)$ has the bounce solution $\phi(r)$, then the potential $V(\chi)=\alpha U(\chi / \beta)$ has the bounce solution $\chi=\beta \phi(\sqrt{\alpha} r / \beta)$. The solution is also invariant under a constant offset of the potential.

We take advantage of these transformations to modify the potential and the field so that the distance between the true and the false vacua is 1 , the value of the potential at the true vacuum is 0 , and the maximum value reached by the potential along the initial path from the true to the false vacuum is 1 . These choices mean that the values of the field and its derivatives that we try to match are all of the same order, which makes it easier to find the solution.

We also change the scale of the variable $\Delta$ [36] by offsetting the $r_{i}$ by $s \Delta$ rather than just $\Delta$. We choose $s$ to be the average of the $r_{i}$ values. This makes the magnitude of $\Delta$ similar to that of the other parameters, even in extreme thin-wall cases with $r_{i} \gg 1$.

\subsection{Readjusting $r$ values}

We used approximate analytic solutions in sections 4.2.1 and 4.2.2. For these approximations to be valid, we need $r_{1}$ small enough and $r_{n}$ large enough so that $\varphi_{1}$ is close to $\varphi_{0}$ and $\varphi_{n}$ close to $\varphi_{\mathrm{fv}}$. While we construct the initial guess (see section 4.6) so that this is true, later improvement of $\varphi_{1}$ and $\varphi_{n}$ may move these values outside the range that allows for an accurate approximation. Conversely, if $\varphi_{1}$ is very close to $\varphi_{0}$ or $\varphi_{n}$ very close to $\varphi_{\mathrm{fv}}$, it means that we can use the analytic solution for larger regions. 
To keep $\varphi_{1}$ and $\varphi_{n}$ in the desirable ranges, we use a constant, $f_{s}$, giving the permissible distance between $\varphi_{0}$ and $\varphi_{1}$ and another, $f_{e}$, giving the permissible distance between $\varphi_{n}$ and $\varphi_{\mathrm{fv}}$, both as a fraction of the distance from $\varphi_{\mathrm{tv}}$ to $\varphi_{\mathrm{fv}}$. Both constants default to 0.01. If the code reaches distances that are larger, we add new $r$ points below the current $r_{1}$ or above the current $r_{n}$ to the points $r_{1}, \ldots, r_{n}$, and if they are much smaller (by factor 10), we remove points from $r_{1}, \ldots r_{n}$ and use the analytic solution over a larger range.

\subsection{Choice of the initial profile}

Probably the most important step in solving a set of equations is choosing an initial point for the solver which is close to the real solution. A bad choice which is out of the basin of attraction would make it impossible to find the solution. When we have more than one field, there are two parts to the initial guess: what path through field space should the initial profile follow, and how should it move along this path as a function of $r$ ?

For the path, by default we simply take a straight line from the true to the false vacuum. But we also allow specification of a set of points $\left\{\varphi_{1}, \ldots, \varphi_{P}\right\}$ through which the initial solution must go. In this case we draw a smooth curve (a cubic spline, or quadratic if $P=1$ ) through the points $\left\{\boldsymbol{\varphi}_{\mathrm{tv}}, \boldsymbol{\varphi}_{1}, \boldsymbol{\varphi}_{2}, \ldots, \boldsymbol{\varphi}_{P}, \boldsymbol{\varphi}_{\mathrm{fv}}\right\}$. We parameterize this path as $\phi_{\mathrm{path}}(\lambda)$ with $\lambda=0 \ldots 1$.

For the shape of the profile as a function of $r$, we use an initial guess based on a thin-wall analogy. We first define a one-dimensional potential

$$
U_{1 \mathrm{~d}}(\lambda)=U\left(\phi_{\mathrm{path}}(\lambda)\right)+\left(3 \lambda^{4}-4 \lambda^{3}\right)\left(U\left(\boldsymbol{\varphi}_{\mathrm{fv}}\right)-U\left(\boldsymbol{\varphi}_{\mathrm{tv}}\right)\right) .
$$

This modified potential has two degenerate minima at $\lambda=0$ and $\lambda=1$ and it shares the same curvature with the original potential at the true vacuum. This potential allows for a domain-wall solution in the form

$$
r(\lambda)=r_{0}+\int \frac{d \lambda}{\sqrt{2 U_{1 \mathrm{~d}}(\lambda)}}\left|\frac{d \phi_{\text {path }}}{d \lambda}\right| .
$$

We can compute the surface tension of this solution,

$$
\sigma=\int d \lambda \sqrt{2 U_{1 \mathrm{~d}}(\lambda)}\left|\frac{d \phi_{\text {path }}}{d \lambda}\right|
$$

and determine the bubble radius in the thin-wall limit by setting the the energy of the bubble wall according to Eq. (4.14) equal to the decrease in energy from the true vacuum inside, giving

$$
r_{\text {thin }}=\frac{(D-1) \sigma}{U\left(\boldsymbol{\varphi}_{\mathrm{fv}}\right)-U\left(\boldsymbol{\varphi}_{\mathrm{tv}}\right)}
$$

We chose the constant of integration $r_{0}$ so that the field at the half-way path length between true and false vacua is at $r_{\text {thin }}$. The initial profile is then defined by inverting (4.13) and using $\phi_{\text {initial }}(r)=\phi_{\text {path }}(\lambda(r))$.

Once we have the profile, we need to choose the points $r_{i}$. We choose $r_{1}$ to be midway from the center to the edge of the region where the analytic approximation can be used, as defined in section 4.5 , and similarly $r_{n}$ midway from the false vacuum to the edge of the final analytic region.

To decide how many $r_{i}$ values to use, we attempt to make the growth of the field during any shooting region no more than a certain limit, by default a factor of 30 . We make the 
approximation of exponential growth as in section 4.1. Thus the $r$ values should be separated by no more than $\delta r_{\max }$ where $e^{\delta r_{\max } \sqrt{B_{\max }}}$ is the allowable growth and $B_{\max }$ the largest eigenvalue of the Hessian matrix. However, since we don't know what values of the field we will use, we find the eigenvalues of the Hessian in the true and false vacua and choose $B_{\max }$ to be the largest of any of these. Having found $\delta r_{\max }$, we divide the range between $r_{1}$ and $r_{n}$ into the smallest number of equal segments that leads to an interval no more than $\delta r_{\max }$.

\subsection{Calculation of the action}

Once we have found the correct profile for the instanton, we would like to find the tunneling action. It is given by

$$
S[\phi]=A_{D-1} \int_{0}^{\infty} d r r^{D-1}\left[\frac{1}{2} \partial_{r} \phi_{i} \partial_{r} \phi_{i}+U(\phi(r))-U\left(\varphi_{\mathrm{fv}}\right)\right]=S_{1}+S_{2},
$$

where $A_{D-1}$ is the area of the unit (D-1)-dimensional sphere, and $S_{1}$ is the kinetic term and $S_{2}$ the rest. Assuming that $\phi(r)$ is a solution to the equations of motion, we can transform (4.16) in several ways. First, consider a family of scale-transformed functions $\phi(\Lambda r)$. We can calculate the action in (4.16) using the profile $\phi(\Lambda r)$, following [37],

$S(\Lambda)=A_{D-1} \int_{0}^{\infty} d r r^{D-1}\left[\frac{1}{2} \partial_{r} \phi_{i}(\Lambda r) \partial_{r} \phi_{i}(\Lambda r)+U(\phi(\Lambda r))-U\left(\varphi_{\mathrm{fv}}\right)\right]=\Lambda^{2-D} S_{1}+\Lambda^{-D} S_{2}$,

This action must have a stationary point at $\Lambda=1$, which implies

$$
D S_{2}=S_{1}(2-D) .
$$

We can also integrate by parts in $S_{1}$. The boundary terms vanish, and we can use the equation of motion (2.5) to find

$$
S_{1}=-\frac{A_{D-1}}{2} \int_{0}^{\infty} d r r^{D-1} \frac{\partial U}{\partial \phi_{i}} \phi_{i}
$$

Our goal is to give, as much as possible, the action of the correct instanton profile, rather than the action of the actual profile that we found (which is divergent, because our profile has small discontinuities where the shooting regions join). So we are free to make any transformations to the action in terms of the exact profile, and then use the approximate profile in the computation.

Computation using $\partial_{r} \phi_{i}$ would be a poor choice, because the derivative is less accurately represented than the function itself in the numerical result of solving the differential equation. We might use (4.18) to write the action in terms of $S_{2}$ alone, but this fails when $D=2$. So instead we use (4.18) to write the action in terms of $S_{1}$ alone, and calculate that with (4.19). As long as the profile can be differentiated analytically, using $\partial U / \partial \phi_{i}$ does not introduce any additional error.

Thus we perform numerically the integral

$$
S[\phi]=-\frac{\pi^{D / 2}}{\Gamma(1+D / 2)} \int_{0}^{\infty} d r r^{D-1}\left[\frac{\partial U}{\partial \phi_{i}}(\phi(r))-\frac{\partial U}{\partial \phi_{i}}\left(\varphi_{\mathrm{fv}}\right)\right] \phi_{i}(r) .
$$

With exact computation, $\partial U / \partial \phi_{i}$ would vanish at the false vacuum. But numerical error can give a tiny result, which we subtract off explicitly. This prevents it from being amplified into an infinite error under the integral out to $r=\infty$. 

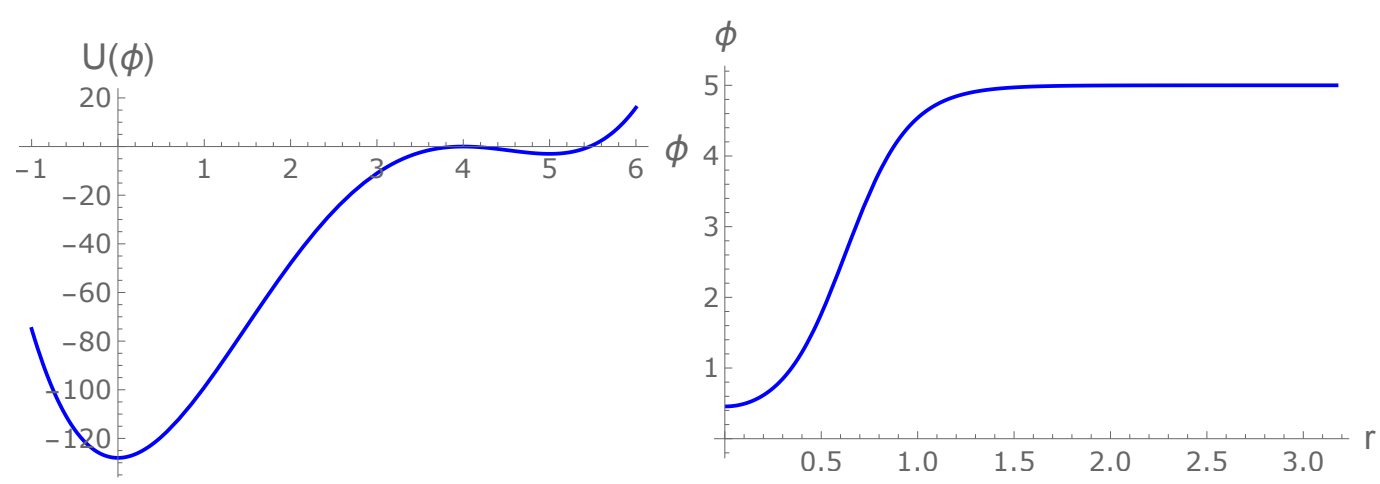

Figure 6. The potential and field profile for the one-dimensional potential given in (5.1). This is an example of a thick-wall solution solved in under four seconds.
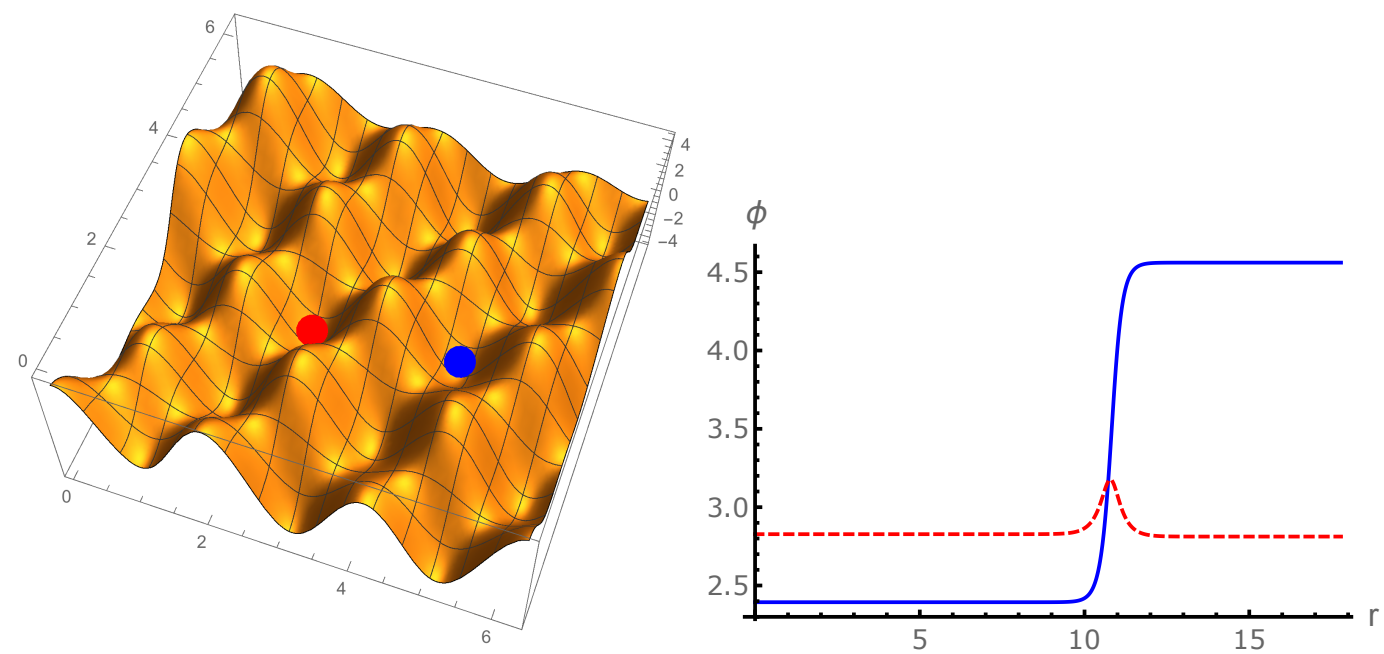

Figure 7. The potential and field profile for the two-dimensional potential given in (5.2). True and false vacua are shown by the red and blue circles. Blue solid and red dashed lines correspond to $\phi_{1}$ and $\phi_{2}$. The true and false vacua are at $(2.39338,2.82768)$ and $(4.56086,2.81235)$.

\section{Examples of potentials solved by the code}

Here we show examples of one, two and four field cases solved using this code. We tried the one-dimensional potential

$$
U(\phi)=\phi^{4}-12 \phi^{3}+40 \phi^{2} .
$$

This potential has minima at $\phi=0$ and $\phi=5$, and the code calculated the bounce in under 4 seconds on a Macbook Pro. The potential and solution are shown in Figure 6.

As a two-dimensional example, we solved the bounce solution for the two-field potential given by

$$
U\left(\phi_{1}, \phi_{2}\right)=\sin \left(\phi_{1}-\phi_{2}\right)+\frac{1}{2} \cos \left(\phi_{1}+\phi_{2}\right)+\cos 3\left(\phi_{1}+\phi_{2}\right)+2 \cos \frac{3}{2}\left(2 \phi_{1}-\phi_{2}\right) .
$$

This was solved in ten seconds on a Macbook Pro. The potential and field profiles are shown in Figure 7. 


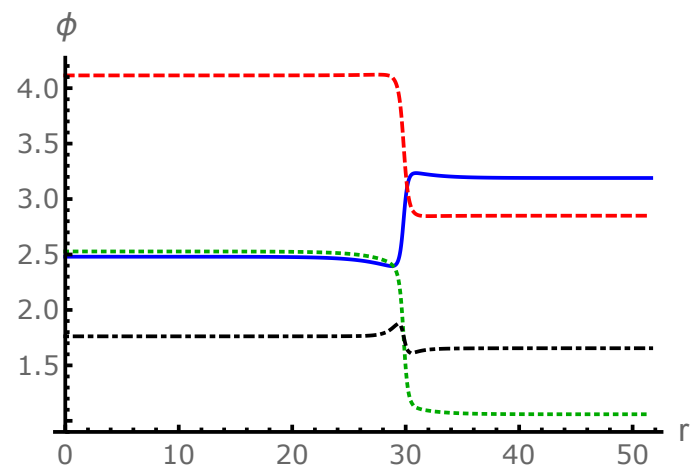

Figure 8. The potential and field profile for the four-dimensional potential given in (5.3). This is an example of a thin-wall solution. The blue solid, red dashed, green dotted and black dot-dashed correspond to $\phi_{1}, \phi_{2}, \phi_{3}$ and $\phi_{4}$.

As the last example we show a four-field case which took about fifteen minutes to get the solution. The potential is given by

$$
\begin{aligned}
U= & -1.2 \cos \left(\phi_{1}+2 \phi_{2}-\phi_{3}-\phi_{4}\right)-1.25 \cos \left(2 \phi_{1}-\phi_{2}-2 \phi_{3}-\phi_{4}\right) \\
& -0.75 \cos \left(\phi_{1}-2 \phi_{2}-2 \phi_{3}-\phi_{4}\right) \\
& -\cos \left(\phi_{1}+\phi_{2}-\phi_{3}+\phi_{4}\right)-0.5 \cos \left(\phi_{1}-\phi_{2}-\phi_{3}-2 \phi_{4}\right) .
\end{aligned}
$$

We found the instanton that gives the tunneling from a false vacuum at $(3.18979,2.84979$, $1.05933,1.65474)$ to a true vacuum at $(2.48005,4.1149,2.52733,1.76197)$. The field profiles are shown in Figure 8.

\section{Quality of the code}

We describe here more systematically the abilities of the code.

\subsection{Range of application}

We have successfully tested our code on random quartic potentials for $N$ up to 9 . We have found bubbles whose radius is larger than the wall thickness by a factor of 200 , and extreme thick wall cases, where there is no clear distinction between the interior and the wall.

For comparison, Kusenko [38] computed actions for 8 fields, while Dasgupta [6] used 10 but computed only a bound on the action. As far as we know no other authors have used more than 3 fields.

\subsection{Success fraction}

We tested our code with potentials in a box of side length $L$. We summed up to 10 Fourier modes randomly chosen among those with with wavelengths down to $L / 6$, with random coefficients chosen from a Gaussian distribution. For each potential we found all minima and calculated the action to tunnel from each minimum to the closest minimum, if that minimum had a smaller energy.

For $N=2$, the code succeeded $96 \%$ of the time in about 10,000 tries, with a typical time about 10 seconds on a Xeon X5675 processor. For $N=3$, the code succeeded in $95 \%$ of about 3,000 tries, with a typical time of about 1 minute. 


\subsection{Speed}

Our code finds solutions in a matter of seconds for one to three fields, minutes for four to seven fields, and hours for eight to nine fields. We have not attempted to run our code for ten or more fields.

Most of the time is spent computing the Jacobian. The Jacobian for each shooting region has $4 N^{2}$ elements. These are determined by integrating a matrix differential equation, as discussed in appendix A, which requires matrix multiplication, and thus $8 N^{3}$ operations for each integration step in the differential equation. One could perhaps abandon explicit calculation of the Jacobian in favor of finite differences, or write a special-purpose program for doing the integration. The code could also be run in parallel, at a minimum by handling the different shooting regions on different processors.

\subsection{Quality of solution}

Our code supports computations with arbitrary precision numbers, so numerical error can be made as small as desired at the price of significantly increased runtime. Unfortunately, there is still an inaccuracy resulting from the initial and final analytic regions, which turns out to be the most significant one. It may be made small by decreasing the parameters $f_{s}$ and $f_{e}$ (defined in section 4.5), but the resulting parameterization of the problem using $\varphi_{1}$ close to $\varphi_{0}$ and $\varphi_{n}$ close to $\varphi_{\mathrm{fv}}$ leads to slow convergence toward the solution. Nevertheless, by comparing solutions with one choice of $f_{s}$ and $f_{e}$ to more accurate solutions with a smaller choice, we can find out how good a result we can get. The default choice of $10^{-2}$ for these parameters gives relative accuracy about $10^{-3}$ in the action, while reducing them to $10^{-3}$ gives relative accuracy about $10^{-5}$.

For comparison, Ref. [12, 38] claimed accuracies of order $10^{-2}$, and [33] claimed profiles accurate to $10^{-3}$. Konstandin \& Huber [35] report errors of $7 \times 10^{-6}$ using 1600 lattice sites.

\subsection{Possible issues}

Here we describe certain difficulties the user may encounter with the code. Some of these may be resolved in future releases.

- Runaway potentials (i.e., those whose true vacua do not exist) are not currently supported. Note that it is always possible to modify such potentials, moving the true vacuum in to finite field values, in a way which does not affect the bounce instanton.

- As discussed in subsection 4.2, near the beginning and end of the profile, we use analytic solutions to the potential expanded to second-order in a Taylor series around $\varphi_{1}$ and $\varphi_{\mathrm{fv}}$, respectively. For this to work, Mathematica has to be able to compute the second derivative, which causes trouble with certain non-analytic functions such as Abs and UnitStep. At the moment the best strategy is to smooth out such jumps by analytic functions such as Tanh. The potential should not have jumps of this kind between $\varphi_{0}$ and $\varphi_{1}$ or between $\varphi_{n}$ and $\varphi_{\mathrm{fv}}$, or the result will be quite inaccurate.

- Potentials whose Hessian has a vanishing eigenvalue at the false vacuum are not supported. A vanishing eigenvalue at a point chosen as $r_{1}$ would also be problematic, but this is unlikely ever to occur unless the Hessian eigenvalue vanishes over a noninfinitesimal region.

- As discussed above, while the accuracy of the solution can be improved by using small values for $f_{i}$ and $f_{s}$, the resulting parameterization leads to much slower convergence. 


\section{Comparison with other methods}

To our knowledge, all techniques for finding instanton solutions involve either shooting or the division of the space of $r$ to be considered into a lattice, i.e., a sequence of fixed $r$ points. Some methods use a hybrid of these two techniques.

In simple shooting, one just varies $\varphi_{0}$ to look for a solution which stays near $\varphi_{\mathrm{fv}}$ for as long as possible. As discussed above, this technique is unstable, but it works fine for thick-wall solutions, where there is not much exponential growth. Even in thin-wall cases, it is possible to beat the instability by the use of high precision arithmetic, but the needed precision can be very high, so this is not an efficient technique. Avoiding such problems by using multiple shooting regions gives the algorithm described in this paper.

Functional minimization problems can be solved by relaxation, in which one creates a discrete approximation to the function and finds the minimum by varying the parameters of this approximation. Unfortunately, the present problem is not minimization of the action, because the action is a maximum with regard to dilation of the profile, and a minimum over all other variations. However, the solution can be found minimizing an improved action [38] or the integral of the square of the deviation from the equations of motion [39, 40].

Alternatively, Refs. $[6,12,33]$ separated out the problem of finding the path taken by the field in field space, which is strictly a minimization problem, and solved the one-dimensional problem of finding the profile by shooting techniques. Refs. [12, 41] discuss a gradient descent/ascent method for finding stationary points which are not minima, but according to Ref. [12], splitting the path and profile problems is much more efficient. Konstandin \& Huber [35] used a lattice equation of motion, which they solved as a Newton's method problem with two equations (field and derivative) for each lattice site.

The difficulty of all such methods is that accurate results require using a large number of points. Ref. [35] reported relative error of $7 \times 10^{-6}$ in the action, but they used 1600 lattice sites to do it. Such methods are especially problematic for a large number of fields $N$, because the set of variables to be operated on is then multiplied by $N$. The multi-shooting method described here is able to achieve good accuracy without a large number of variables. ${ }^{2}$

\section{Conclusion}

We have presented here a multi-shooting method for the calculation of instantons for the nucleation of bubbles of true vacuum inside a false vacuum in a potential depending on several fields. This method succeeds nearly all the time in the tests that we have done. For one to three fields it runs quite quickly, and even for nine fields it succeeds, albeit slowly.

We have made our Mathematica implementation of this method freely available, as described in appendix $\mathrm{C}$ below. We hope it will be useful, both for the calculation of tunneling rates in specific models, and for statistical surveys of large numbers of random potentials.

\section{Acknowledgments}

We thank Brian Greene, Alex Vilenkin, Erick Weinberg, and I-Sheng Yang for helpful discussions and comments. This work was supported in part by the National Science Foundation

\footnotetext{
${ }^{2}$ Of course this method works by solving differential equations, which means that functions are evaluated at some sequence of points. However, the runtime grows only linearly with the number of points used in differential equation solving, whereas other equation-solving or minimization procedures normally have much faster growth with the number of variables.
} 
under grants 1213888 and 1518742.

\section{A Calculation of Jacobian}

In order to implement Powell's hybrid method we need to know how the equations change when we change the parameters. One way to do so is by changing the parameters by small amounts to see how the result changes. In fact, Powell [42] gives a procedure to do this, primarily using the sequence of parameter choices. However, direct calculation of the Jacobian is more reliable and less prone to numerical errors, and it is possible to do that here.

\section{A.1 Solving for Jacobian along the path}

To compute the Jacobian, we need to know, for each shooting step, the way in which the solution to the differential equation, and thus the value of the field and its derivative at the endpoint, depend on the initial conditions.

We start with a system of first order differential equations,

$$
\mathbf{f}^{\prime}(r)=\mathbf{F}(r, \mathbf{f}(r))
$$

with the initial conditions $\mathbf{f}(0)$ given. That is, $\mathbf{f}(r)$ is a vector function of $r$ whose derivative depends upon $r$ and the value of $\mathbf{f}$ at $\mathrm{r}$. We want to know how the solution of the differential equation at some point $r$ depends on changes to the initial conditions. So suppose that we start with infinitesimally different initial conditions $\mathbf{g}(0)$. Assuming $\mathbf{F}$ is a smooth function, these new conditions will give rise to an infinitesimally different solution $\mathbf{g}(r)$ and we can define $\mathbf{A}(r)=\mathbf{g}(r)-\mathbf{f}(r)$. Then $\mathbf{A}$ satisfies a simple differential equation,

$$
\mathbf{A}^{\prime}(r)=\mathbf{g}^{\prime}(r)-\mathbf{f}^{\prime}(r)=\mathbf{F}(r, \mathbf{g})-\mathbf{F}(r, \mathbf{f})=\mathbf{F}(r, \mathbf{f}+\mathbf{A})-\mathbf{F}(r, \mathbf{f})=\frac{\partial \mathbf{F}}{\partial f_{j}} A_{j}(r) .
$$

The first-order effect on $f_{i}(r)$ due to changes in $f_{i}(0)$ is given by the Jacobian matrix with components

$$
J_{i j}=\frac{\partial f_{i}(r)}{\partial f_{j}(0)} .
$$

To find such a component, we choose $A_{j}(0)=1$ for the $j$ of interest and 0 for the others, and then $J_{i j}(r)=A_{i}(r)$. Thus, for each $j$, the $J_{i j}(r)$ are the solution to the system of differential equations

$$
J_{i j}^{\prime}(r)=\frac{\partial F_{i}}{\partial f_{k}} J_{k j}(r) \quad \text { with initial conditions } J_{l j}(0)=\delta_{l j} .
$$

Since (A.4) holds for each $j$, we can consider it to be a matrix differential equation giving all components of $J_{i j}(r)$.

Let us apply this technique to the Jacobian for the differential equations given in (2.5). First we convert these equations into a set of first order equations by defining auxiliary fields $\phi_{i}^{\prime}=\phi_{i+N}$ and rewriting $(2.5)$ as

$$
\begin{aligned}
\phi_{i+N}^{\prime} & =-\frac{D-1}{r} \phi_{i+N}+\frac{\partial U}{\partial \phi_{j}}, \\
\phi_{i}^{\prime} & =\phi_{i+N} .
\end{aligned}
$$


Now the problem is mapped into the form of (A.1) and we get a simple expression

$$
\frac{\partial F_{A}}{\partial f_{B}}=\left(\begin{array}{c|c}
\mathbf{0} & \mathbf{I} \\
\hline \partial^{2} U / \partial \phi_{i} \partial \phi_{j} & -\frac{D-1}{r} \mathbf{I}
\end{array}\right)
$$

To calculate the Jacobian we first solve the differential equations (2.5) and then evaluate (A.6) along the solutions $\phi$ to solve (A.4) and get $J_{A B}(r)$.

For the first and last interval we must also take account of the fact that we specify only $\phi_{1}$ and $\phi_{n}$, and $\phi_{1}^{\prime}$ and $\phi_{n}^{\prime}$ are determined from these. Those derivatives then in turn determine the initial conditions used in shooting. Thus the Jacobian for these cases must include the effect of a change in the field value upon the derivative found by the procedures of section 4.2 .

When we consider variations of $\phi_{1}$, we should consider changes to the Hessian matrix $B_{i j}$ of equation (4.2), because of being evaluated at a different $\boldsymbol{\phi}$. But these depend on the third derivatives of the potential and are complicated to calculate. So we have ignored this effect in computing the Jacobian. This should not creating any larger problem than ignoring these same third derivatives in computing the analytic approximations.

\section{A.2 Jacobian with respect to $\Delta$}

As described in subsection 4.3 we do not evaluate the fields at constant values of $r$. Instead we have an extra variable $\Delta$ which changes the value of the $r_{i}$ where we apply the parameters. Fortunately, we do not need to solve extra differential equations to compute the effect of changing $\Delta$. The matching points are

$$
R_{i}=\left\{r_{1}+\Delta, r_{2}+\Delta, \ldots r_{n}+\Delta\right\}
$$

Here we show the procedure for case of $n=4$ case, which is depicted in figure 4 , with a single field. Generalization to arbitrary $n$ and $N$ is straightforward. First we calculate the following Jacobian using the technique described in section A.1:

$$
J=\left(\begin{array}{cccc}
\frac{\partial \phi_{L}\left(r_{2}\right)}{\partial \phi\left(r_{1}\right)} & 0 & 0 & 0 \\
\frac{\partial \phi_{L}^{\prime}\left(r_{2}\right)}{\partial \phi\left(r_{1}\right)} & 0 & 0 & 0 \\
0 & \frac{\partial \phi_{L}\left(r_{3}\right)}{\partial \phi\left(r_{2}\right)} & \frac{\partial \phi_{L}\left(r_{3}\right)}{\partial \phi^{\prime}\left(r_{2}\right)} & -\frac{\partial \phi_{R}\left(r_{3}\right)}{\partial \phi\left(r_{4}\right)} \\
0 & \frac{\partial \phi_{L}^{\prime}\left(r_{3}\right)}{\partial \phi\left(r_{2}\right)} & \frac{\partial \phi_{L}^{\prime}\left(r_{3}\right)}{\partial \phi^{\prime}\left(r_{2}\right)} & -\frac{\partial \phi_{R}^{\prime}\left(r_{3}\right)}{\partial \phi\left(r_{4}\right)}
\end{array}\right) .
$$

When we add the new variable $\Delta$, our Jacobian will the same number of rows, but one more column, giving the dependence of the various parameters on $\Delta$,

$$
J_{\text {total }}=\left(\begin{array}{l|c}
J & \frac{\partial \phi_{L}\left(r_{2}\right)}{\partial \Delta} \\
\frac{\partial \phi_{L}^{\prime}\left(r_{2}\right)}{\partial \Delta} \\
\frac{\partial \phi_{L}\left(r_{3}\right)}{\partial \Delta}-\frac{\partial \phi_{R}\left(r_{3}\right)}{\partial \Delta} \\
\frac{\partial \phi_{L}^{\prime}\left(r_{3}\right)}{\partial \Delta}-\frac{\partial \phi_{R}^{\prime}\left(r_{3}\right)}{\partial \Delta}
\end{array}\right) .
$$

There are two types of effects due to $\Delta$. First, after we solve the differential equation, changing $r_{i}$ changes the place at which the solution is evaluated. Second, changing $r_{i}$ changes which solution of the differential equation we use when we start from the same initial data $\phi\left(r_{i}\right)$ and $\phi^{\prime}\left(r_{i}\right)$. We can propagate the new data back to the former location of $r_{i}$, changing the $\phi$ and $\phi^{\prime}$ values used as initial conditions appropriately. 
In all, we find six types of terms that we need to calculate:

$$
\begin{aligned}
& \frac{\partial \phi_{L}\left(r_{3}\right)}{\partial \Delta}=\phi_{L}^{\prime}\left(r_{3}\right)-\phi^{\prime}\left(r_{2}\right) \frac{\partial \phi_{L}\left(r_{3}\right)}{\partial \phi\left(r_{2}\right)}-\phi^{\prime \prime}\left(r_{2}\right) \frac{\partial \phi_{L}\left(r_{3}\right)}{\partial \phi^{\prime}\left(r_{2}\right)} \\
& \frac{\partial \phi_{L}^{\prime}\left(r_{3}\right)}{\partial \Delta}=\phi_{L}^{\prime \prime}\left(r_{3}\right)-\phi^{\prime}\left(r_{2}\right) \frac{\partial \phi_{L}^{\prime}\left(r_{3}\right)}{\partial \phi\left(r_{2}\right)}-\phi^{\prime \prime}\left(r_{2}\right) \frac{\partial \phi_{L}^{\prime}\left(r_{3}\right)}{\partial \phi^{\prime}\left(r_{2}\right)} \\
& \frac{\partial \phi_{L}\left(r_{2}\right)}{\partial \Delta}=\phi_{L}^{\prime}\left(r_{2}\right)-\phi^{\prime}\left(r_{1}\right) \frac{\partial \phi_{L}\left(r_{2}\right)}{\partial \phi\left(r_{1}\right)} \\
& \frac{\partial \phi_{L}^{\prime}\left(r_{2}\right)}{\partial \Delta}=\phi_{L}^{\prime \prime}\left(r_{2}\right)-\phi^{\prime}\left(r_{1}\right) \frac{\partial \phi_{L}^{\prime}\left(r_{2}\right)}{\partial \phi\left(r_{1}\right)} \\
& \frac{\partial \phi_{R}\left(r_{3}\right)}{\partial \Delta}=\phi_{R}^{\prime}\left(r_{3}\right)-\phi^{\prime}\left(r_{4}\right) \frac{\partial \phi_{R}^{\prime}\left(r_{3}\right)}{\partial \phi\left(r_{4}\right)} \\
& \frac{\partial \phi_{R}^{\prime}\left(r_{3}\right)}{\partial \Delta}=\phi_{R}^{\prime \prime}\left(r_{3}\right)-\phi^{\prime}\left(r_{4}\right) \frac{\partial \phi_{R}^{\prime}\left(r_{3}\right)}{\partial \phi\left(r_{4}\right)}
\end{aligned}
$$

The partial derivatives on the right in (A.10) are all elements of the original Jacobian $J$. The first derivatives at $r_{2}$ and $r_{3}$ are variables, while those at $r_{1}$ and $r_{4}$ can be computed from the analytic forms given in section 4.2. Finally, the second derivatives can be computed using the equations of motion,

$$
\phi^{\prime \prime}\left(r_{i}\right)=U^{\prime}\left(\phi\left(r_{i}\right)\right)-\frac{D-1}{r_{i}} \phi^{\prime}\left(r_{i}\right) .
$$

\section{B Powell's method for solving equations}

M. J. D. Powell [42] describes a method for solving multiple simultaneous equations that is a hybrid of Newton's method and gradient descent. We describe this method briefly here; for more details, see Ref. [42].

Powell's hybrid method attempts to iteratively find the simultaneous root of a set of nonlinear functions $f_{i}\left(x_{1}, \ldots\right)$. Given some current guess $\mathbf{x}$, the method finds some step $\boldsymbol{\delta}$ such that $\mathbf{x}+\boldsymbol{\delta}$ is a better approximation to the desired root. This is done by (potentially) combining two possible steps.

We first linearize the equations around the point $\mathbf{x}$. Newton's method works by considering next the solution to these linearized equations. This method is quadratically convergent if one is close enough to the actual solution. But if not, the next guess can be much further from the actual solution than the previous one.

An alternative is to move in the direction which most rapidly decreases the squared error $F(\mathbf{x})=\sum_{i} x_{i}^{2}$. This is a quadratic in the linearized $f_{i}(\mathbf{x})$, so it achieves a minimum at some distance from $\mathbf{x}$ and we will call this direction and distance the gradient step.

Powell's hybrid method combines these ideas as follows. It maintains at each time a desired step size $\Delta$ (in the Euclidean norm on the space of parameter values). If the step recommended by Newton's method is shorter than $\Delta$. Powell's method tries to take the Newton step. If not, consider the gradient step. If it is longer than $\Delta$, try going distance $\Delta$ in the gradient direction. If it is shorter, try a linear combination of the gradient step and the Newton step that has length $\Delta$.

In all cases, if the trial step decreases $F$ we take it, and adjust the step size based on how much progress we are making. If the trial fails to decrease $F$, we try shorter and shorter steps until we find one that does. If only an infinitesimal step decreases $F$, it appears that we have found a local minimum and must give up. 
The method starts always with a gradient step. If the method appears to be working, it moves more and more toward Newton's method, and eventually converges rapidly on the solution. If things are going poorly, it slowly descends the surface of $F$ until it finds an area where it can move rapidly towards the solution (or fails at a local minimum).

In order to apply this method, we need the Jacobian $\partial f_{i} / \partial x_{j}$. Powell [42] describes techniques for maintaining an approximate Jacobian by a series of function evaluations. But here we are able to compute the Jacobian numerically, as described in appendix A, so we do not need these techniques. As described in section 4.3, we extended the method to allow more variables than functions. This has no effect at all on the gradient method: we move in the direction that most rapidly decreases $F$, just as before. For Newton's method, there are now many possible steps that solve the linearized equations, and we choose the shortest of these.

\section{Obtaining and running the code}

We have implemented the above procedures in Mathematica. The code can be downloaded from http://cosmos.phy.tufts. edu/AnyBubble/. There is a manual which explains how to find instanton solutions and all the options available in the code.

\section{References}

[1] J. S. Langer, Statistical theory of the decay of metastable states, Annals Phys. 54 (1969) $258-275$.

[2] A. J. Leggett, A theoretical description of the new phases of liquid He-3, Rev. Mod. Phys. 47 (1975) 331-414.

[3] N. Cabibbo, L. Maiani, G. Parisi and R. Petronzio, Bounds on the Fermions and Higgs Boson Masses in Grand Unified Theories, Nucl. Phys. B158 (1979) 295-305.

[4] P. Q. Hung, Vacuum Instability and New Constraints on Fermion Masses, Phys. Rev. Lett. 42 (1979) 873.

[5] J. Elias-Miro, J. R. Espinosa, G. F. Giudice, G. Isidori, A. Riotto and A. Strumia, Higgs mass implications on the stability of the electroweak vacuum, Phys. Lett. B709 (2012) 222-228, [1112.3022].

[6] I. Dasgupta, Estimating vacuum tunneling rates, Phys. Lett. B394 (1997) 116-122, [hep-ph/9610403].

[7] U. Sarid, Tools for tunneling, Phys. Rev. D58 (1998) 085017, [hep-ph/9804308].

[8] P. J. Steinhardt, Relativistic Detonation Waves and Bubble Growth in False Vacuum Decay, Phys. Rev. D25 (1982) 2074.

[9] E. Witten, Cosmic Separation of Phases, Phys. Rev. D30 (1984) 272-285.

[10] C. J. Hogan, Gravitational radiation from cosmological phase transitions, Mon. Not. Roy. Astron. Soc. 218 (1986) 629-636.

[11] A. Kosowsky, M. S. Turner and R. Watkins, Gravitational waves from first order cosmological phase transitions, Phys. Rev. Lett. 69 (1992) 2026-2029.

[12] J. M. Cline, G. D. Moore and G. Servant, Was the electroweak phase transition preceded by a color broken phase?, Phys. Rev. D60 (1999) 105035, [hep-ph/9902220].

[13] L. Randall and G. Servant, Gravitational waves from warped spacetime, JHEP 05 (2007) 054, [hep-ph/0607158]. 
[14] V. A. Kuzmin, V. A. Rubakov and M. E. Shaposhnikov, On the Anomalous Electroweak Baryon Number Nonconservation in the Early Universe, Phys. Lett. B155 (1985) 36.

[15] E. Witten, Instability of the Kaluza-Klein Vacuum, Nucl. Phys. B195 (1982) 481-492.

[16] D. J. Gross, M. J. Perry and L. G. Yaffe, Instability of Flat Space at Finite Temperature, Phys. Rev. D25 (1982) 330-355.

[17] A. H. Guth, Eternal inflation and its implications, J. Phys. A40 (2007) 6811-6826, [hep-th/0702178].

[18] A. Vilenkin, A Measure of the multiverse, J. Phys. A40 (2007) 6777, [hep-th/0609193].

[19] R. Bousso and J. Polchinski, Quantization of four form fluxes and dynamical neutralization of the cosmological constant, JHEP 06 (2000) 006, [hep-th/0004134].

[20] L. Susskind, The Anthropic landscape of string theory, hep-th/0302219.

[21] A. Aazami and R. Easther, Cosmology from random multifield potentials, JCAP 0603 (2006) 013, [hep-th/0512050].

[22] S. Sarangi, G. Shiu and B. Shlaer, Rapid Tunneling and Percolation in the Landscape, Int. J. Mod. Phys. A24 (2009) 741-788, [0708.4375].

[23] S. H. H. Tye, A Renormalization Group Approach to the Cosmological Constant Problem, 0708.4374 .

[24] Q.-G. Huang and S. H. H. Tye, The Cosmological Constant Problem and Inflation in the String Landscape, Int. J. Mod. Phys. A24 (2009) 1925-1962, [0803.0663].

[25] B. Greene, D. Kagan, A. Masoumi, D. Mehta, E. J. Weinberg et al., Tumbling through a landscape: Evidence of instabilities in high-dimensional moduli spaces, Phys.Rev. D88 (2013) 026005, [1303.4428].

[26] A. Aravind, B. S. DiNunno, D. Lorshbough and S. Paban, Analyzing multifield tunneling with exact bounce solutions, Phys. Rev. D91 (2015) 025026, [1412.3160].

[27] M. Dine and S. Paban, Tunneling in Theories with Many Fields, JHEP 10 (2015) 088, [1506.06428].

[28] A. Masoumi and A. Vilenkin, Vacuum statistics and stability in axionic landscapes, JCAP 1603 (2016) 054, [1601.01662].

[29] I. Yu. Kobzarev, L. B. Okun and M. B. Voloshin, Bubbles in Metastable Vacuum, Sov. J. Nucl. Phys. 20 (1975) 644-646.

[30] S. R. Coleman, The Fate of the False Vacuum. 1. Semiclassical Theory, Phys.Rev. D15 (1977) 2929-2936.

[31] A. D. Linde, Decay of the False Vacuum at Finite Temperature, Nucl. Phys. B216 (1983) 421.

[32] A. Bayliss, A double shooting scheme for certain unstable and singular boundary value problems, Mathematics of Computation 32 (1978) 61.

[33] C. L. Wainwright, CosmoTransitions: Computing Cosmological Phase Transition Temperatures and Bubble Profiles with Multiple Fields, Comput. Phys. Commun. 183 (2012) 2006-2013, [1109.4189].

[34] D. D. Morrison, J. D. Riley and J. F. Zancanaro, Multiple shooting method for two-point boundary value problems, Commun. ACM 5 (Dec., 1962) 613-614.

[35] T. Konstandin and S. J. Huber, Numerical approach to multi dimensional phase transitions, JCAP 0606 (2006) 021, [hep-ph/0603081].

[36] K. D. Olum and A. Masoumi, Auxiliary variables for nonlinear equations with softly broken symmetries, 1611.03950. 
[37] G. H. Derrick, Comments on nonlinear wave equations as models for elementary particles, J. Math. Phys. 5 (1964) 1252-1254.

[38] A. Kusenko, P. Langacker and G. Segre, Phase transitions and vacuum tunneling into charge and color breaking minima in the MSSM, Phys. Rev. D54 (1996) 5824-5834, [hep-ph/9602414].

[39] J. M. Moreno, M. Quiros and M. Seco, Bubbles in the supersymmetric standard model, Nucl. Phys. B526 (1998) 489-500, [hep-ph/9801272].

[40] P. John, Bubble wall profiles with more than one scalar field: A Numerical approach, Phys. Lett. B452 (1999) 221-226, [hep-ph/9810499].

[41] J. M. Cline, J. R. Espinosa, G. D. Moore and A. Riotto, String mediated electroweak baryogenesis: A Critical analysis, Phys. Rev. D59 (1999) 065014, [hep-ph/9810261].

[42] M. J. D. Powell, A hybrid method for nonlinear equations, in Numerical methods for nonlinear algebraic equations (P. Rabinowitz, ed.), ch. 6, pp. 87-114. Gordon and Breach Science Publishers, New York, 1970. 